\title{
Chronic Diarrhea as a Presenting Manifestation of Common Variable Immunodeficiency
}

\author{
Amir Hossein Hosseini ${ }^{1}$, Mehrnaz Mesdaghi $^{2,}$, , Ali Akbar Sayyari ${ }^{2}$, Zahra Chavoshzadeh $^{3}$ \\ ${ }^{1}$ Shohadaye Tajrish Hospital, Shahid Beheshti University of Medical Science, Tehran, IR Iran \\ 2 Mofid Children's Hospital, Shahid Beheshti University of Medical Sciences, Tehran, IR Iran \\ 3 Pediatric Infections Research Center, Shahid Beheshti University of Medical Sciences, Tehran, IR Iran \\ *Corresponding author: Mehrnaz Mesdaghi, Department of Immunology and Allergy, Mofid Children's Hospital, Shahid Beheshti University of Medical Sciences, Tehran, IR Iran. Tel: \\ +98-2122227035, Fax:+98-2122220254, E-mail: mehrnaz_mesdaghi@yahoo.com.
}

Received: May 18, 2013; Revised: Jun 20, 2013; Accepted: July 04, 2013

\begin{abstract}
Common variable immunodeficiency (CVID) is one of the primary immunodeficiencies, which usually presents with recurrent bacterial infections, particularly in respiratory and gastrointestinal systems and hypogammaglobulinemia. We present here a case of CVID who was suffering from chronic watery diarrhea since 3 months before admission. Past medical history was uneventful about recurrent infections. No abnormality was revealed on physical examination. After thorough investigations about other possible causes of chronic diarrhea, and based on laboratory data of hypogammaglobuliemia, we considered him as a case of CVID with an isolated manifestation.

Keywords: Common Variable Immunodeficiency; Diarrhea; T cell immunodeficiency Primary
\end{abstract}

\section{Introduction}

Common variable immunodeficiency (CVID) is one of the prevalent primary immunodeficiencies, with heterogeneous manifestations, mostly reduction in immunoglobulin levels and recurrent bacterial infections. We present here a case of CVID with the rare presentation of chronic non-infectious diarrhea, without concomitant recurrent infections.

\section{Case}

This 3-year-old boy was admitted to our tertiary pediatric hospital due to a history of 3 months refractory watery diarrhea. One-kilogram weight loss, since beginning of the current illness was detected. His parents were not relatives. His past medical history about growth and development was acceptable and he was not suffering from chronic or recurrent infections (e.g. draining otitis media, refractory sinusitis, pneumonia, etc.). According to national immunization program, his vaccination history was complete and uneventful. On physical examination, he was ill but not toxic and his vital signs were in normal range for his age. Growth indices were not affected, except weight for age that was below 10th percentile. Examination of buccal cavity revealed mild thrush. Tonsils were visible. No lymphadenopathy or hepatosplenomegaly was detected. Other physical examinations were normal. In laboratory data, complete blood count and biochemistry showed normal parameters. Erythrocyte sedimentation rate was reported $34 \mathrm{~mm} / \mathrm{h}$. Stool examination showed the presence of undigested material and fat; with pH 5.5, but ova, cyst or trophozoites was not seen. Stool culture was negative. Tuberculin skin test showed 3 millimeters induration. Sweat chloride test was normal. The child was seronegative for HIV, HBsAg, and $\mathrm{HCV}$, and HIV PCR was reported negative twice. Serum protein electrophoresis showed a normal protein analysis, except reduction of gamma globulins. Autoimmune markers were checked and all of them were within normal range. Evaluation for immunodeficiency disorders showed very low levels of IgA, IgM, and IgG (Table 1). Antitetanus and anti-diphtheria IgG were measured and they were 0.006 and $0.003 \mathrm{IU} / \mathrm{mL}$, respectively $(<0.1$ is considered non responsive). His blood typing showed O-blood group and his isohemmaglutinin titers were less than $1 / 4$. The results of flow cytometric lymphocyte subsets analysis are shown in (Table 2). Dihydrorudamine (DHR) test was not in favor of chronic granulomatous disease. Colonoscopy and tissue biopsy were done that showed non-specific colitis. Finally, the CVID was established according to the European society for immunodeficiencies diagnostic criteria (1). He was treated with intravenous antibiotics and intravenous immunoglobulin infusions. Diarrhea was stopped and an acceptable weight gain was

Implication for health policy/practice/research/medical education: This article helps pediatricians for diagnosis of CVID.

Copyright @ 2014, Pediatric Infections Research Center; Licensee Kowsar. Ltd. This is an Open Access article distributed under the terms of the Creative Commons Attribution License (http://creativecommons.org/licenses/by/3.0), which permits unrestricted use, distribution, and reproduction in any medium, provided the original work is properly cited. 
achieved after starting intravenous immunoglobulin administration.

\begin{tabular}{lll}
\hline \multicolumn{2}{l}{ Table 1. Nephelometric Immunoglobin Analysis Report } \\
\hline Immunoglobulin & Patient & Normal Ranges \\
\hline IgG & 0.149 & $0.5-13 \mathrm{~g} / \mathrm{L}$ \\
IgM & Non-detectable & $0.34-2.55 \mathrm{~g} / \mathrm{L}$ \\
IgE & 0.1 & Up to $68 \mathrm{IU} / \mathrm{mL}$ \\
Ig A & Non-detectable & $0.19-2.2 \mathrm{~g} / \mathrm{L}$ \\
\hline
\end{tabular}

Table 2. Flow Cytometric Lymphocyte Subset Analyses, Cell Count and Subpopulations

\begin{tabular}{ll}
\hline Cell Count and Subpopulation & Result \\
\hline White Blood cell Count & $8800 / \mathrm{mm}^{3}$ \\
\hline Neutrophil & $40 \%$ \\
Lymphocyte & $53 \%$ \\
CD3 & $80.5 \%$ \\
\hline CD4 & $10.9 \%$ \\
CD8 & $61.3 \%$ \\
\hline CD56 & $8.9 \%$ \\
CD19 & $9.9 \%$ \\
\hline CD4/CD8 & 0.18 \\
\hline
\end{tabular}

\section{Discussion}

CVID is a diverse group of disorders, which arise from B and $\mathrm{T}$ lymphocytes dysfunctions and results in hypogammaglobulinemia (2). CVID has been defined clinically by the presence of recurrent infections and a reduction in IgG (at least 2 SD below the mean), and at least one other immunoglobulin isotypes, as well as by a failure to mount a significant specific antibody response to challenge with vaccination or natural infections $(1,3)$. CVID usually presents with recurrent sinopulmonary bacterial infections as a result of poor antibody responses and decreased serum immunoglobulins. Infections of the lower respiratory tracts (recurrent pneumonia) often result in bronchiectasis (4). Recurrent respiratory infections affect up to $98 \%$ of CVID patients (5). Gastrointestinal manifestations are the presenting symptom of CVID in about $3 \%$ of the patients (6). Totally, the gastrointestinal tract is affected in about half of the CVID patients. The major gastrointestinal manifestations of CVID are transient or persistent diarrhea, reported in $21-57 \%$ of subjects. Other intestinal presentations include intestinal malabsorbtion, chronic giardiasis and amebiasis, and atrophic gastritis with pernicious anemia. About $20 \%$ of patients with CVID have gut symptoms without an infectious cause (5-9). Although gastrointestinal manifestations are common findings in CVID, they are rare presenting signs and symptoms (as the first presentation) in CVID, especially without concomitant respiratory and gastrointestinal infections. Similarly, in our patient gut infection was never documented on repeated stool examinations and he had never experienced respiratory infections or any other recurrent infections before diagnosis. Actually, chronic non-infectious diarrhea was the single manifestation of CVID in this patient, which is a really rare presentation of CVID. Pater et al. have also recently reported a case of CVID with the rare presentation of chronic diarrhea. They also couldn't find an infectious origin for his diarrhea, but different from our report, their patient had also previous recurrent infections (10). Another report by Onbasi et al., discusses a 39-year-old patient with CVID, presenting with chronic diarrhea, but giardiasis was found to be the reason of diarrhea. She was also suffering from recurrent infections since childhood (11).

In a study by Aghamohammadi et al. (9), infection, super infection, ulcerative colitis, food-sensitive enteropathy, autoimmune enteropathy, and celiac disease are considered the causes of diarrhea and malabsorption manifestation in these patients.

It is important to consider hypogammaglobulinemia in any patient with a history of chronic diarrhea and patients should undergo a full assessment of immune system including measurement of serum immunoglobulin levels, antibody function evaluation, and B and T-cell subsets enumeration.

The mainstays of treatment for CVID remain replacement immunoglobulin (Ig) and antibiotics for infections and appropriate treatment for the non-infectious complications $(7,9)$.

\section{Acknowledgements}

There is no acknowledgment.

\section{Authors' Contribution}

Amir Hossein Hosseini: Patient's physician and writing the manuscript; Mehrnaz Mesdaghi: Diagnosis, revising and submitting the manuscript; Ali Akbar Sayyari: Patient's physician; Zahra Chavoshzadeh: Diagnosis and patient's physician.

\section{Financial Disclosure}

The authors declare that there is no conflict of interest.

\section{Funding/Support}

This research/article has not been supported by any institution.

\section{References}

1. Yong PF, Thaventhiran JE, Grimbacher B. "A rose is a rose is a rose," but CVID is Not CVID common variable immune deficiency (CVID), what do we know in 2011? Adv Immunol. 2011;111:47-107.

2. Yong PF, Tarzi M, Chua I, Grimbacher B, Chee R. Common variable 
immunodeficiency: an update on etiology and management. Immunol Allergy Clin North Am. 2008;28(2):367-86.

3. Hermaszewski RA, Webster AD. Primary hypogammaglobulinaemia: a survey of clinical manifestations and complications. Q J Med. 1993;86(1):31-42.

4. Teahon K, Webster AD, Price AB, Weston J, Bjarnason I. Studies on the enteropathy associated with primary hypogammaglobulinaemia. Gut. 1994;35(9):1244-9.

5. Cunningham-Rundles C, Bodian C. Common variable immunodeficiency: clinical and immunological features of 248 patients. Clin Immunol.1999;92(1):34-48.

6. Quinti I, Soresina A, Spadaro G, Martino S, Donnanno S, Agostini C, et al. Long-term follow-up and outcome of a large cohort of patients with common variable immunodeficiency. J Clin Immunol. 2007;27(3):308-16.

7. Quinti I, Soresina A, Spadaro G, Martino S, Donnanno S, Agostini $\mathrm{C}$, et al. Long-term follow-up and outcome of a large cohort of patients with common variable immunodeficiency. J Clin Immunol. 2007;27(3):308-16.
8. Aghamohammadi A, Farhoudi A, Nikzad M, Moin M, Pourpak $\mathrm{Z}$, Rezaei N, et al. Adverse reactions of prophylactic intravenous immunoglobulin infusions in Iranian patients with primary immunodeficiency. Ann Allergy Asthma Immunol. 2004;92(1):60-4.

9. Aghamohammadi A, Farhoudi A. Use of immunoglobulin in patients with antibody deficiencies. In: Farhoudi A, editors. Immunodeficiencies in Iran.Tehran, Iran: Shayan Nemoodar; 1997. p. 277-307.

10. Aghamohammadi A, Farhoudi A, Moin M, Rezaei N, Kouhi A Pourpak Z, et al. Clinical and immunological features of 65 Iranian patients with common variable immunodeficiency. Clin Diagn Lab Immunol. 2005;12(7):825-32.

11. Patel J, Kumar A, Agasti A, Choksey A, Phadke A, Sawant P. CVID enteropathy- a rare cause of chronic diarrhea in a child. Indian J Pediatr. 2012;79(10):1374-6.

12. Onbasi K, Gunsar F, Sin AZ, Ardeniz O, Kokuludag A, Sebik F. Common variable immunodeficiency (CVID) presenting with malabsorption due to giardiasis. Turk J Gastroenterol. 2005;16(2):111-3. 